\title{
Spectral approximations for characteristic roots of delay differential equations
}

\author{
C. P. Vyasarayani · S. Subhash · T. Kalmár-Nagy
}

Received: 2 October 2013 / Revised: 13 January 2014 / Accepted: 27 January 2014 / Published online: 22 February 2014

(C) Springer-Verlag Berlin Heidelberg 2014

\begin{abstract}
In this paper we develop approximations to the characteristic roots of delay differential equations using the spectral tau and spectral least squares approach. We study the influence of different choices of basis functions in the spectral solution on the numerical convergence of the characteristic roots. We found that the spectral tau method performed better than the spectral least squares method. Legendre and Chebyshev bases provide much better convergence properties than the mixed Fourier basis.
\end{abstract}

Keywords Delay $\cdot$ Spectrum $\cdot$ Spectral least squares . Spectral-tau

\section{Introduction}

Delays are inherent in many natural and physical processes, for example our ability to locate the direction of the sound source comes from the capacity of our ears to detect the small time lag (delay) between the sound perceived by our left and right ear [1]. In engineering, DDEs are used as mathematical models in analyzing manufacturing processes [2], real-time sub-structuring [3], and in control theory [4].

Stability analysis of DDEs is important, particularly to find parameters for which the physical process is stable or to estimate the largest delay that a system can tolerate to remain stable. In machine tool vibrations, for example, the

C. P. Vyasarayani $\cdot$ S. Subhash

Department of Mechanical Engineering, Indian Institute of Technology Hyderabad, Ordnance Factory Estate, Medak 502205, Andhra Pradesh, India

T. Kalmár-Nagy ( $\varangle)$

Mitsubishi Electric Research Laboratory, 201 Broadway,

Cambridge, MA 02139, USA

e-mail: kalmar-nagy@merl.com tool dynamics are governed by DDEs [5] and with the help of stability analysis we can find machining parameters for decreased surface roughness.

DDEs are infinite dimensional systems, inasmuch as their characteristic equations have infinitely many roots. The Lambert $\mathrm{W}$ function [6-8], Laplace transforms [9], and Dsubdivision methods [10] can be used to study stability of DDEs with single delay. Asymptotics can be used to calculate roots of scalar DDEs with few delays [11]. Lyapunov functions [12-14] can also be used to determine the stability of DDEs. General methods for obtaining the stability of DDEs with multiple delays first convert the DDE into a partial differential equation (PDE) [15-18] with a linear boundary condition. Then, ordinary differential equations (ODEs) based approximation for the PDE can be developed by using spatial discretization methods, such as:

- Semi-discretization [19]

- Spectral least squares [20]

- Spectral tau methods [15,21]

- Pseudo-spectral collocation [22-24]

- Time finite elements $[25,26]$

- Continuous time approximation [27,28]

- Finite difference methods [17,29]

As we represent a DDE using a PDE with a linear boundary condition, the way in which we incorporate the boundary condition while developing the ODE approximations will influence the spectrum (roots of the characteristic equation of the DDE). For example the boundary condition can be incorporated using spectral-tau method [15,21], or by spectral-least squares [20] method.

In this paper we compare the spectral-tau and spectralleast squares approaches for obtaining the characteristic roots of DDEs. Spectral methods are advantageous due to their 
exponential convergence rates [30] to the actual solution. Previously, within the frame work of spectral (Galerkin) methods, only mixed Fourier basis was used for obtaining characteristic roots or solution of the DDEs [20,21]. The convergence characteristics of the mixed Fourier basis have not been explored in detail, for example in [21] for a test problem (see Table 1) only one root converges to a tolerance of two decimal places when using mixed Fourier basis in the Galerkin solution. In this paper we study the influence of the choice of spectral basis, i.e, shifted Chebyshev, shifted Legendre, and mixed Fourier basis on the convergence of the characteristic roots for some example problems.

\section{Recasting and solving a delay equation as an advection equation}

For clarity of presentation we only consider a scalar DDE in this work. The theory developed in this paper can be easily extended to higher order DDEs following the work of Vyasarayani [20]. We consider the scalar delay equation with $m$ delays

$\dot{x}(t)+a x(t)+\sum_{q=1}^{m} b_{q} x\left(t-\tau_{q}\right)=0, \quad \tau_{q}>0$.

The initial function is specified as

$x(t)=\theta(t),-\tau \leq t \leq 0$,

where $\tau=\max \left(\tau_{1}, \tau_{2}, \ldots, \tau_{m}\right)$. By introducing the socalled shift of time $y(s, t)=x(t+s), s \in[-\tau, 0)$, the initial value problem (Eqs. (1), (2)) can be recast into the following initial-boundary value problem for the advection Eqs. $[16,17]$

$\frac{\partial y(s, t)}{\partial t}=\frac{\partial y(s, t)}{\partial s}, \quad s \in[-\tau, 0]$,

$\left.\frac{\partial y(s, t)}{\partial t}\right|_{s=0}=-a y(0, t)-\sum_{q=1}^{m} b_{q} y\left(-\tau_{q}, t\right)$,

$y(s, 0)=\theta(s), \quad s \in[-\tau, 0]$.

We discuss two methods, the spectral-tau method and spectral-least squares method for the approximate solution of Eqs. (3-5).

\subsection{Spectral-tau method}

In the spectral-tau method we assume a solution to the PDE (Eq. 3) of the following form:

$y(s, t)=\sum_{i=1}^{\infty} \phi_{i}(s) \eta_{i}(t)$, where $\phi_{i}(s)$ are the basis functions and $\eta_{i}(t)$ are the time dependent coordinates. For practical reasons the sum is terminated at $N$ terms, i.e.

$y(s, t)=\boldsymbol{\phi}(s)^{T} \boldsymbol{\eta}(t)$,

where $\phi(s)=\left[\phi_{1}(s), \phi_{2}(s), \ldots, \phi_{N}(s)\right]^{T}$ and $\eta(t)=$ $\left[\eta_{1}(t), \eta_{2}(t), \ldots, \eta_{N}(t)\right]^{T}$. Substituting the series solution Eq. (7) in Eq. (3) we get (the symbol' denotes derivative with respect to $s$ )

$\boldsymbol{\phi}(s)^{T} \dot{\boldsymbol{\eta}}(t)=\boldsymbol{\phi}^{\prime}(s)^{T} \boldsymbol{\eta}(t)$.

Pre-multipling Eq. (8) with $\phi(s)$ and integrating over the domain we get:

$\int_{-\tau}^{0} \boldsymbol{\phi}(s) \boldsymbol{\phi}(s)^{T} d s \dot{\boldsymbol{\eta}}(t)=\int_{-\tau}^{0} \boldsymbol{\phi}(s) \boldsymbol{\phi}^{\prime}(s)^{T} d s \boldsymbol{\eta}(t)$.

In matrix form

$\mathbf{A} \dot{\boldsymbol{\eta}}(t)=\mathbf{B} \boldsymbol{\eta}(t)$,

with

$\mathbf{A}=\int_{-\tau}^{0} \boldsymbol{\phi}(s) \boldsymbol{\phi}(s)^{T} d s$

$\mathbf{B}=\int_{-\tau}^{0} \boldsymbol{\phi}(s) \boldsymbol{\phi}^{\prime}(s)^{T} d s$.

Substituting Eq. (7) in Eq. (4) we get the scalar equation

$\boldsymbol{\phi}(0)^{T} \dot{\boldsymbol{\eta}}(t)=\left[-a \boldsymbol{\phi}(0)^{T}-\sum_{q=1}^{m} b_{q} \boldsymbol{\phi}\left(-\tau_{q}\right)^{T}\right] \boldsymbol{\eta}(t)$.

Note that $(10,13)$ provide $N+1$ independent equations. To arrive at a determinate system we truncate the system (10) and augment it with (13) to form

$\mathbf{M}_{\text {Tau }} \dot{\boldsymbol{\eta}}(t)=\mathbf{K}_{\text {Tau }} \boldsymbol{\eta}(t)$,

where

$\mathbf{M}_{\text {Tau }}=\left[\begin{array}{c}\overline{\mathbf{A}} \\ \boldsymbol{\phi}(0)^{T}\end{array}\right]$,

$\mathbf{K}_{\text {Tau }}=\left[\begin{array}{c}\overline{\mathbf{B}} \\ \left.-a \boldsymbol{\phi}(0)^{T}-\sum_{q=1}^{m} b_{q} \phi\left(-\tau_{q}\right)^{T}\right],\end{array}\right.$

and matrices $\overline{\mathbf{A}}, \overline{\mathbf{B}}$ are obtained by deleting the last row of matrix $\mathbf{A}$ and $\mathbf{B}$, respectively. The initial conditions for Eq. (14) is $\boldsymbol{\eta}(0)=\mathbf{M}^{-1} \int_{-\tau}^{0} \boldsymbol{\phi}(s) \theta(s) d s$ and the solution of the DDE can be obtained as $x(t)=y(0, t)=\boldsymbol{\phi}(0)^{T} \boldsymbol{\eta}(t)$. The finite dimensional system (14) represents an approximation for Eq. (1). 


\subsection{Spectral least-squares method}

The error in the PDE (3) due to the substitution of the truncated approximate solution $y(s, t)=\sum_{i=1}^{N} \phi_{i}(s) \eta_{i}(t)$ is

$e(s, t)=\boldsymbol{\phi}(s)^{T} \dot{\eta}(t)-\boldsymbol{\phi}^{\prime}(s)^{T} \eta(t)$.

A good approximation is characterized by a "small" error $e(s, t)$ subject to the boundary constraint Eq. (13). To minimize the error, we aim to solve the following constrained optimization problem:

$$
\begin{aligned}
& \min _{\dot{\eta}(t)} \frac{1}{2} \int_{-\tau}^{0} e(s, t)^{2} d s \\
& \quad=\min _{\dot{\boldsymbol{\eta}}(t)} \frac{1}{2} \int_{-\tau}^{0}\left[\boldsymbol{\phi}(s)^{T} \dot{\boldsymbol{\eta}}(t)-\boldsymbol{\phi}^{\prime}(s)^{T} \boldsymbol{\eta}(t)\right]^{2} d s \\
& \text { s.t. } \boldsymbol{\phi}(0)^{T} \dot{\boldsymbol{\eta}}(t)=\left[-a \boldsymbol{\phi}(0)^{T}-\sum_{q=1}^{m} b_{q} \boldsymbol{\phi}\left(-\tau_{q}\right)^{T}\right] \boldsymbol{\eta}(t),
\end{aligned}
$$

i.e. we are interested to find $\dot{\eta}(t)$ such that the integral of the square of the error function over the domain is minimized. We introduce a Lagrange multiplier $\Lambda$ and construct the following Lagrangian

$$
\begin{aligned}
L(\dot{\boldsymbol{\eta}}(t), \Lambda)= & \frac{1}{2} \int_{-\tau}^{0}\left[\boldsymbol{\phi}(s)^{T} \dot{\boldsymbol{\eta}}(t)-\boldsymbol{\phi}^{\prime}(s)^{T} \boldsymbol{\eta}(t)\right]^{2} d s \\
& -\Lambda\left[\boldsymbol{\phi}(0)^{T} \dot{\boldsymbol{\eta}}(t)+a \boldsymbol{\phi}(0)^{T} \boldsymbol{\eta}(t)\right. \\
& \left.+\sum_{q=1}^{m} b_{q} \boldsymbol{\phi}\left(-\tau_{q}\right)^{T} \boldsymbol{\eta}(t)\right] .
\end{aligned}
$$

We seek to minimize $L(\dot{\eta}(t), \Lambda)$. The first order optimality conditions for the minimization of $L$ are [31]

$$
\begin{gathered}
\frac{\partial L}{\partial \dot{\boldsymbol{\eta}}(t)}=0, \\
\frac{\partial L}{\partial \Lambda}=0 .
\end{gathered}
$$

Substituting Eq. (20) in Eqs. (21) and (22) we get:

$$
\begin{aligned}
& \mathbf{A} \dot{\eta}(t)=\mathbf{B} \eta(t)+\boldsymbol{\phi}(0) \Lambda, \\
& \boldsymbol{\phi}(0)^{T} \dot{\eta}(t)=-a \boldsymbol{\phi}(0)^{T} \eta(t)-\sum_{q=1}^{m} b_{q} \boldsymbol{\phi}\left(-\tau_{q}\right)^{T} \boldsymbol{\eta}(t),
\end{aligned}
$$

where $\mathbf{A}$ and $\mathbf{B}$ are the basis-dependent matrices defined in Eqs. (11) and (12) respectively.
Solving Eqs. (23) and (24) for the Lagrange multiplier yields

$$
\begin{aligned}
\Lambda= & -\frac{\boldsymbol{\phi}(0)^{T} \mathbf{A}^{-1} \mathbf{B}}{\boldsymbol{\phi}(0)^{T} \mathbf{A} \boldsymbol{\phi}(0)} \boldsymbol{\eta}(t) \\
& -\frac{1}{\boldsymbol{\phi}(0)^{T} \mathbf{A}^{-1} \boldsymbol{\phi}(0)}\left(a \boldsymbol{\phi}(0)^{T}+\sum_{q=1}^{m} b_{q} \boldsymbol{\phi}\left(-\tau_{q}\right)^{T}\right) \boldsymbol{\eta}(t) .
\end{aligned}
$$

Substituting the value of $\Lambda$ in Eq. (23) and simplifying we get:

$\mathbf{A} \dot{\boldsymbol{\eta}}(t)=\mathbf{K}_{L S} \boldsymbol{\eta}(t)$,

where

$$
\begin{aligned}
\mathbf{K}_{L S}= & \mathbf{B}-\frac{1}{\boldsymbol{\phi}(0)^{T} \mathbf{A}^{-1} \boldsymbol{\phi}(0)}\left(\boldsymbol{\phi}(0) \boldsymbol{\phi}(0)^{T} \mathbf{A}^{-1} \mathbf{B}\right. \\
& \left.+a \boldsymbol{\phi}(0) \boldsymbol{\phi}(0)^{T}+\sum_{q=1}^{m} b_{q} \boldsymbol{\phi}(0) \boldsymbol{\phi}\left(-\tau_{q}\right)^{T}\right) .
\end{aligned}
$$

Equation (26) represents the ODE approximation of the DDE Eq. (1).

\section{Computing spectra}

Substituting $x(t)=c e^{\lambda t}$ in Eq. (1) we obtain the characteristic equation $(\mathcal{C}(\lambda)$ is the characteristic function)

$\mathcal{C}(\lambda)=\lambda+a+\sum_{q=1}^{m} b_{q} e^{-\lambda \tau_{q}}=0$.

A complex root $\lambda$ of the characteristic equation (eigenvalue of Eq. 1) is written as

$\lambda=\alpha+i \beta$.

Using Euler's identity in (28) and separating the real and imaginary parts yields

$\alpha+a+\sum_{q=1}^{m} b_{q} e^{-\alpha \tau_{q}} \cos \left(\beta \tau_{q}\right)=0$,

$\beta-\sum_{q=1}^{m} b_{q} e^{-\alpha \tau_{q}} \sin \left(\beta \tau_{q}\right)=0$.

Equations $(30,31)$ are transcendental (exponential quasipolynomials) and have infinitely many roots. We define the spectrum of (1) as the set of roots of the characteristic equation, i.e.

$S=\left\{\lambda_{i} \mid \mathcal{C}\left(\lambda_{i}\right)=0, \operatorname{Re} \lambda_{1} \geq \operatorname{Re} \lambda_{2} \geq ..\right\}$. 
The approximate spectrum obtained by calculating the eigenvalues of the $N \times N$ system Eq. (14) is defined as

$$
\begin{aligned}
\hat{S}_{\text {Tau }}= & \left\{\hat{\lambda}_{i} \mid \operatorname{det}\left(\mathbf{M}_{\text {Tau }} \hat{\lambda}_{i}-\mathbf{K}_{\text {Tau }}\right)=0,\right. \\
& \left.\operatorname{Re} \hat{\lambda}_{1} \geq \operatorname{Re} \hat{\lambda}_{2} \geq . .\right\}
\end{aligned}
$$

and for Eq. (26) as

$\hat{S}_{L S}=\left\{\hat{\lambda}_{i} \mid \operatorname{det}\left(\mathbf{A} \hat{\lambda}_{i}-\mathbf{K}_{L S}\right)=0, \operatorname{Re} \hat{\lambda}_{1} \geq \operatorname{Re} \hat{\lambda}_{2} \geq ..\right\}$.

The error in the $k$-th eigenvalue is defined as

$\varepsilon_{k}=\left|\mathcal{C}\left(\hat{\lambda}_{k}\right)\right|$

The accuracy of the rightmost $R$ roots of the approximate spectrum $\hat{S}$ is characterized by the "tolerance"

$T(R)=\max _{1 \leq k \leq R} \varepsilon_{k}$

We expect the choice of basis functions $\phi(s)$ in Eq. (7) to play an important role in the convergence of the eigenvalues. To test this hypothesis we consider three different basis functions: mixed Fourier basis [21]

$\boldsymbol{\phi}(s)=\left[1, s, \sin \left(\frac{\pi}{\tau} s\right), \sin \left(2 \frac{\pi}{\tau} s\right) \ldots\right]^{T}$,

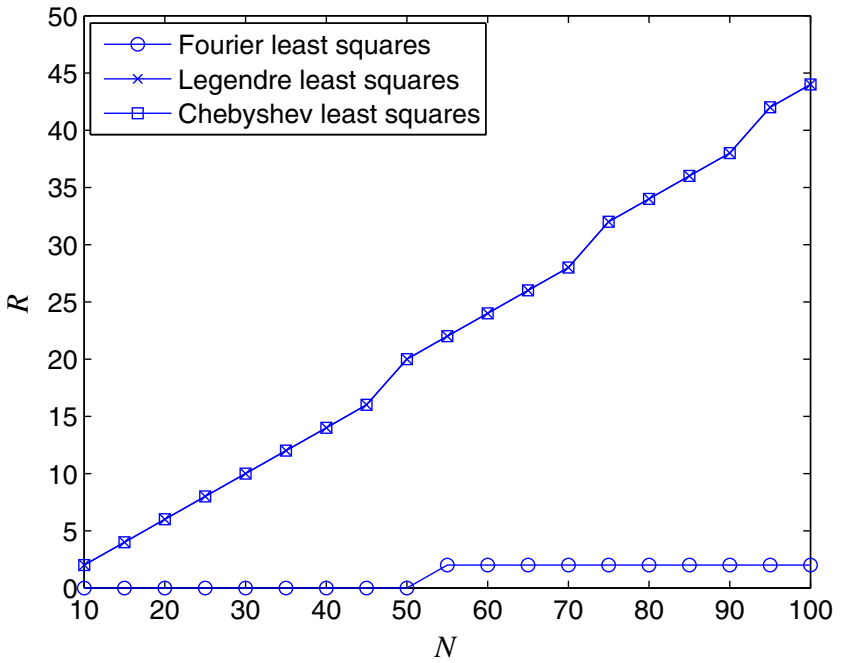

(a)

Fig. 1 Number of converged roots for tolerance $T(R)=10^{-4}$ for increasing $N$ : a spectral least squares method and $\mathbf{b}$ spectral tau method. The parameters are $\tau_{1}=1$ and $b_{1}=1$. The $y$-axis shows the num- shifted Legendre polynomials [30]

$$
\begin{aligned}
\phi_{1}(s)= & 1, \phi_{2}(s)=1+\frac{2 s}{\tau}, \\
\phi_{i}(s)= & \frac{(2 i-3) \phi_{2}(s) \phi_{i-1}(s)-(i-2) \phi_{i-2}(s)}{} \\
& i-1, i=3, \ldots,
\end{aligned}
$$

and shifted Chebyshev polynomials [30]

$$
\begin{aligned}
& \phi_{1}(s)=1, \phi_{2}(s)=1+\frac{2 s}{\tau}, \\
& \phi_{i}(s)=2 \phi_{2}(s) \phi_{i-1}(s)-\phi_{i-2}(s), i=3, \ldots
\end{aligned}
$$

\section{Results}

Here we compare the spectral tau and spectral least squares methods introduced in Sects. 2.1 and 2.2 on equations containing one, two, and (for a good measure) 30 delays. We use mixed Fourier (Eq. 37), Legendre (Eq. 38), and Chebyshev (Eq. 39) basis functions to study convergence properties of the two methods.

We first consider the equation with one time delay

$\dot{x}+b_{1} x\left(t-\tau_{1}\right)=0$.

It is an important case, since the eigenvalues of its characteristic equation

$\lambda+b_{1} e^{-\lambda \tau_{1}}=0$

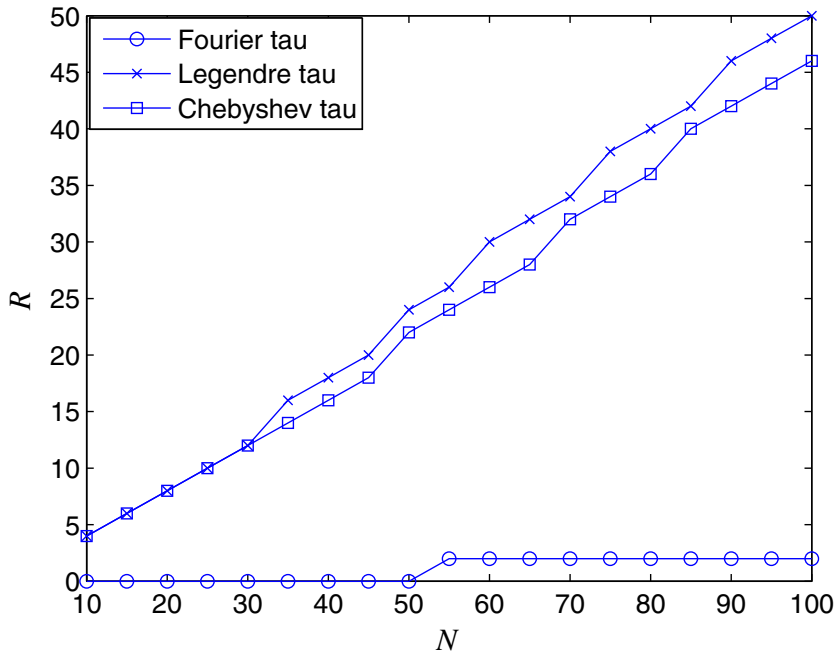

(b)

ber of roots $(R)$ that has converged within a tolerance of $10^{-4}$. The $x$-axis shows the number of terms used in the series solution (Eq. 7) for obtaining the roots 
can be obtained in closed form in terms of Lambert W function [6] as

$\lambda_{r}=\frac{1}{\tau_{1}} W_{r}\left(-b_{1} \tau_{1}\right), r=-\infty, \ldots,-1,0,1, \ldots \infty$.

Here $W_{k}$ corresponds to $k$ th branch of the Lambert W function.

Figure 1 shows the number of converged roots for tolerance $T(R)=10^{-4}$ for increasing $N$ (number of terms used in the series solution (Eq. 7). Here $R$ stands for the number of roots that have converged within a tolerance of $10^{-4}$ (up to four decimal places). As expected, increasing the number of terms in the series solution will yield more and more eigenvalues. For example, with $N=100,44$ roots converge within a tolerance of $10^{-4}$ for Legendre least square and Chebyshev least square method. However, with Fourier least square method only 2 roots converged. In spectral tau method for $N=100,50$ roots converged with Legendre basis and 46 roots converges with Chebyshev basis and only 2 roots converges with mixed Fourier basis. It is surprising to see the poor performance of the mixed Fourier basis for both the tau and the least squares method. Figure $2 \mathrm{a}-\mathrm{c}$ show spectra of Eq. (41) obtained through Eq. (42) for the case $b_{1}=\tau_{1}=1$ (other parameter values yield similar results). Superimposed on the graph are the roots obtained by the Legendre tau method for $N=25$ (Fig. 2a), $N=50$ (Fig. 2b), and $N=100$ (Fig. 2c). We again see that for increasing $N$ more and more eigenvalues of the approximate spectrum $\hat{S}$ (Eq. 33) converge to the exact eigenvalues.

After having established the positive influence of increasing $N$ on convergence, we used $N=25$ to obtain the rest of the results.

Figure 3 shows the average error in each eigenvalue $\varepsilon_{k}$ (see Eq. 35) for 1,000 simulations for spectral tau method and for spectral least squares method. The delay $\tau_{1}$ and $b_{1}$ were randomly selected from uniform distributions between $\tau_{1} \in$ $[0.1,1]$ and $b_{1} \in[-10,10]$ respectively. We can observe

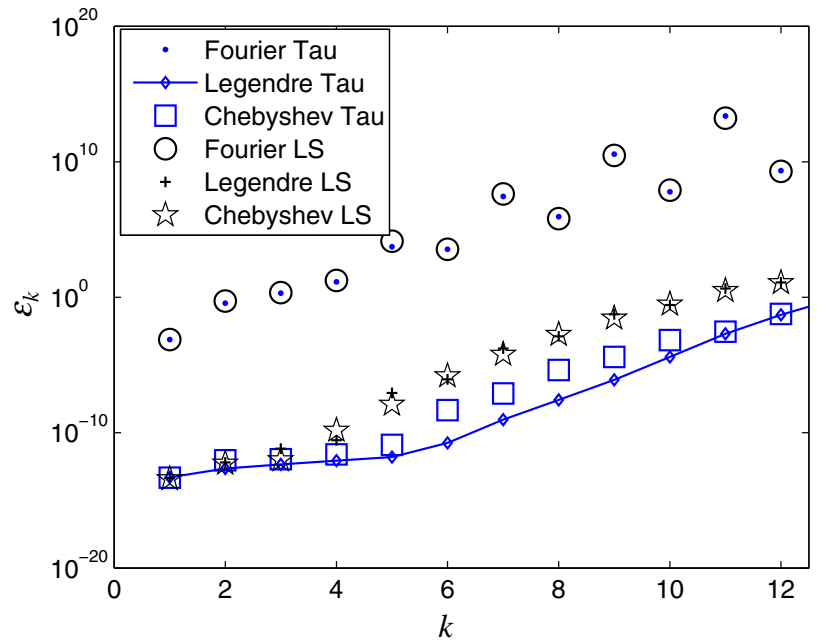

Fig. 3 Average error in the roots for spectral least squares and spectral tau methods with $N=25$. The results are generated using 1,000 Monte Carlo simulations with $b_{1}$ and $\tau_{1}$ selected from a uniform distribution on $[0.1,1]$ and $[-10,10]$, respectively

from Fig. 3 that on an average for a tolerance $10^{-10}, 6$ roots converged with Legendre tau method, 5 roots converged with chebyshev tau method, 4 roots with Legendre least square method, 3 roots with Chebyshev least square method and no roots converged with Fourier tau or Fourier least square method. We see from Fig. 3 that the Legendre tau method has the best performance in terms of number of converged roots.

We also studied the total number of roots $R$ that converge to the required tolerance of $10^{-4}$. To cover a large number of test cases, 10,000 simulations were performed with randomly selected parameters from uniform distributions for a two-delay equation $\left(a \in[-10,10], b_{1} \in[-10,30], b_{2} \in\right.$ $[-10,50], \tau_{1} \in[0.1,5.1]$, and $\left.\tau_{2} \in[0.1,10.1]\right)$ and the equation with 30 delays $\left(a \in[-10,10], \tau_{k} \in[0.1,10.1]\right.$, $\left.b_{k} \in[-10,50], k=1,2, \ldots, 30\right)$. The results are summarized in Table 1 and Table 2 . In the table each entry corre-

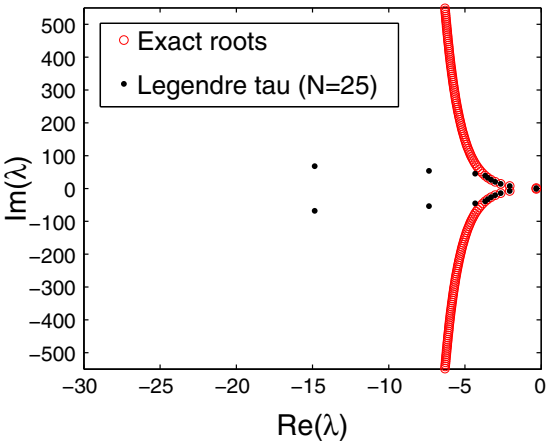

(a)

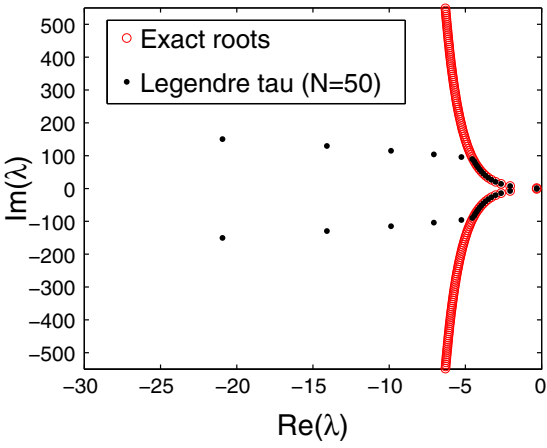

(b)

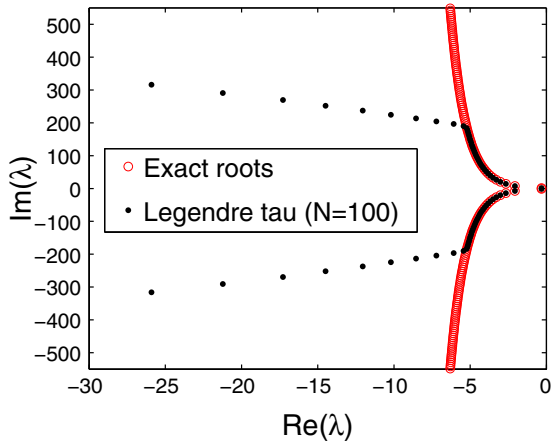

(c)

Fig. 2 Comparison of exact roots obtained with Lambert $\mathrm{W}$ function with approximate roots obtained from Legendre tau method for a $N=25$, b $N=50$ and $\mathbf{c} N=100$. The parameters are $\tau_{1}=1$ and $b_{1}=1$ 
Table 1 Percentage of number of converged roots for different methods and for different basis functions

\begin{tabular}{lrrrrrrrrrrrrr}
\hline $\mathrm{R}$ & 0 & 1 & 2 & 3 & 4 & 5 & 6 & 7 & 8 & 9 & 10 \\
\hline Fourier least squares & 92 & 8 & 0 & 0 & 0 & 0 & 0 & 0 & 0 & 0 & 0 \\
Legendre least squares & 0 & 0 & 0 & 0 & 0 & 0 & 1 & 7 & 69 & 10 & 13 & 0 \\
Chebyshev least squares & 0 & 0 & 0 & 0 & 0 & 0 & 1 & 7 & 70 & 11 & 12 & 0 \\
Fourier tau & 78 & 15 & 4 & 1 & 1 & 0 & 0 & 0 & 0 & 0 & 0 & 0 \\
Legendre tau & 0 & 0 & 0 & 0 & 0 & 0 & 0 & 3 & 27 & 15 & 54 & 1 \\
Chebyshev tau & 0 & 0 & 0 & 0 & 0 & 0 & 0 & 3 & 27 & 15 & 53 & 1 \\
\hline
\end{tabular}

The results are obtained by performing 10,000 Monte Carlo simulations with parameters taken from uniform distributions for two-delay equation $\left(a \in[-10,10], b_{1} \in[-10,30], b_{2} \in[-10,50], \tau_{1} \in[0.1,5.1]\right.$, and $\left.\tau_{2} \in[0.1,10.1]\right)$

Table 2 Percentage of number of converged roots for different methods and for different basis functions

\begin{tabular}{|c|c|c|c|c|c|c|c|c|c|c|c|c|}
\hline $\mathrm{R}$ & 0 & 1 & 2 & 3 & 4 & 5 & 6 & 7 & 8 & 9 & 10 & 11 \\
\hline Fourier least squares & 100 & 0 & 0 & 0 & 0 & 0 & 0 & 0 & 0 & 0 & 0 & 0 \\
\hline Legendre least squares & 0 & 0 & 0 & 0 & 0 & 0 & 37 & 8 & 48 & 2 & 4 & 0 \\
\hline Chebyshev least squares & 0 & 0 & 0 & 0 & 0 & 0 & 37 & 7 & 48 & 2 & 4 & 0 \\
\hline Fourier tau & 100 & 0 & 0 & 0 & 0 & 0 & 0 & 0 & 0 & 0 & 0 & 0 \\
\hline Legendre tau & 0 & 0 & 0 & 0 & 1 & 0 & 39 & 8 & 47 & 2 & 3 & 0 \\
\hline Chebyshev tau & 0 & 0 & 0 & 0 & 0 & 1 & 39 & 8 & 46 & 2 & 3 & 0 \\
\hline
\end{tabular}

The results are obtained by performing 10,000 Monte Carlo simulations with parameters taken from uniform distributions for 30-delay equation $\left(a \in[-10,10], \tau_{k} \in[0.1,10.1], b_{k} \in[-10,50], k=1,2, . ., 30\right)$

sponds to the percentage of times the particular root has converged within a tolerance of $10^{-4}$. For example for Fourier least square method (Table 1), no root converged for $92 \%$ of cases and one root converged for $8 \%$ of total cases.

It is clear that the choice of the mixed Fourier basis has an adverse effect on the number of eigenvalues found. We also note that the expected number of "correct" eigenvalues in the 30-delay case is smaller than that of the two-delay case, but this might be due to the different range of parameters selected for the simulations.

\section{Discussion and conclusions}

It is worth investigating why the mixed Fourier basis performs badly in terms of convergence compared to shifted Legendre and shifted Chebyshev basis (see Fig. 3). In the mixed Fourier basis, we have $\phi(s)=[1, s, \sin (\pi s / \tau), \ldots$, $\sin ((N-2) \pi s / \tau)]^{T}$ and we can see that only the first two terms have non-zero values at $s=-\tau$ and for $s=0$ only one term remains. The information about the rightmost delay appears in the spectral matrices $\mathbf{M}_{T a u}, \mathbf{K}_{\text {Tau }}$ and $\mathbf{K}_{L S}$ through a term like $\phi(-\tau)^{T} \eta(t)$, so the coupling terms for the largest delay are not strong (as most of the terms in $\phi(s)^{T}$ are zeros). This is not the case with the Legendre and Chebysev bases, where $\phi(s)^{T}$ is fully populated. We thus conjecture that the sparse nature of $\phi(s)^{T}$ at $s=0$ and $s=-\tau$ is a (if not the) reason for the bad convergence (see Fig. 3) behavior for the mixed Fourier basis.
We have studied the spectral-tau method and spectral-least squares method for obtaining characteristic roots of a linear DDE. We found that the spectral tau method performed better in terms of number of converged roots for given $N$ (see Fig. 3; Tables 1, 2) than the spectral least squares method. Also the Legendre or Chebyshev basis performed much better (see Fig. 3; Tables 1,2) in terms of error convergence compared to mixed Fourier basis. The spectral-tau method is easy to code and understand, also it performs better than the spectral-least squares method, so we recommend spectral-tau method for analyzing the stability of linear DDEs.

Acknowledgments CPV thanks DST for financial support through the fast track scheme for young scientists (ref: SB/FTP/ETA-0462/2012).

\section{References}

1. Blauert J (1997) Spatial hearing: the psychophysics of human sound localization. MIT Press, MA

2. Balachandran B (2001) Nonlinear dynamics of milling processes. Philos Trans R Soc Lond Ser A Math Phys Eng Sci 359(1781): 793-819

3. Wallace MI, Sieber J, Neild SA, Wagg DJ, Krauskopf B (2005) Stability analysis of real-time dynamic substructuring using delay differential equation models. Earthq Eng Struct Dyn 34(15): $1817-1832$

4. Richard JP (2003) Time-delay systems: an overview of some recent advances and open problems. Automatica 39(10):1667-1694

5. Insperger T, Stépán G (2000) Stability of the milling process. Mech Eng 44(1):47-57 
6. Asl FM, Ulsoy AG (2003) Analysis of a system of linear delay differential equations. J Dyn Syst Meas Control 125(2):215-223

7. Jarlebring E, Damm T (2007) The lambert w function and the spectrum of some multidimensional time-delay systems. Automatica 43(12):2124-2128

8. Yi S, Nelson PW, Ulsoy AG (2010) Time-delay systems: analysis and control using the lambert $\mathrm{W}$ function. World Scientific, New Jersey

9. Kalmár-Nagy T (2009) Stability analysis of delay-differential equations by the method of steps and inverse laplace transform. Differ Equ Dyn Syst 17(1-2):185-200

10. Olgac N, Sipahi R (2002) An exact method for the stability analysis of time-delayed linear time-invariant (LTI) systems. IEEE T Automat Control 47(5):793-797

11. Wahi P, Chatterjee A (2005) Asymptotics for the characteristic roots of delayed dynamic systems. ASME J Appl Mech 72(4):475483

12. Gu K, Chen J, Kharitonov VL (2003) Stability of time delay systems. Springer, New York

13. Gu K, Niculescu SI (2006) Stability analysis of time-delay systems: A lyapunov approach. In advanced topics in control systems theory. Springer, London

14. Fridman Emilia (2001) New Lyapunov-Krasovskii functionals for stability of linear retarded and neutral type systems. Syst Control Lett 43(4):309-319

15. Ito K, Teglas R (1986) Legendre-tau approximations for functionaldifferential equations. SIAM J Control Optim 24(4):737-759

16. Bellen A, Maset S (2000) Numerical solution of constant coefficient linear delay differential equations as abstract cauchy problems. Numer Math 84(3):351-374

17. Koto T (2004) Method of lines approximations of delay differential equations. Comput Math Appl 48(1):45-59

18. Breda D (2006) Solution operator approximations for characteristic roots of delay differential equations. Appl Numer Math 56(3):305317
19. Insperger T, Stépán G (2011) Semi-discretization for time-delay systems. Springer, New York

20. Vyasarayani CP (2012) Galerkin approximations for higher order delay differential equations. ASME J Comput Nonlin Dyn 7(3):031004

21. Wahi P, Chatterjee A (2005) Galerkin projections for delay differential equations. J Dyn Syst Meas Control 127(1):80-87

22. Butcher EA, Ma H, Bueler E, Averina V, Szabo Z (2004) Stability of linear time-periodic delay-differential equations via chebyshev polynomials. Int J Numer Meth Eng 59(7):895-922

23. Breda D, Maset S, Vermiglio R (2005) Pseudospectral differencing methods for characteristic roots of delay differential equations. SIAM J Sci Comput 27(2):482-495

24. Wu Z, Michiels W (2012) Reliably computing all characteristic roots of delay differential equations in a given right half plane using a spectral method. J Comput Appl Math 236(9):2499-2514

25. Mann BP, Patel BR (2010) Stability of delay equations written as state space models. J Vib Control 16(7-8):1067-1085

26. Khasawneh FA, Mann BP (2011) A spectral element approach for the stability of delay systems. Int J Numer Meth Eng 87(6):566-592

27. Sun JQ (2009) A method of continuous time approximation of delayed dynamical systems. Commun Nonlinear Sci Numer Simul 14(4):998-1007

28. Song B, Sun JQ (2011) Lowpass filter-based continuous-time approximation of delayed dynamical systems. J Vib Control 17(8):1173-1183

29. Engelborghs K, Roose D (2002) On stability of LMS methods and characteristic roots of delay differential equations. SIAM J Numer Anal 40(2):629-650

30. Boyd JP (2001) Chebyshev and fourier spectral methods. Dover, Mineola

31. Luenberger DJ (2003) Linear and nonlinear programming. Springer, New York 